\title{
Probing Properties and Structure of Complex Oxides Superlattices using Scanning Electron Nanodiffraction
}

Roberto dos Reis ${ }^{1}$, Weibing Yang ${ }^{2}$, Ravini U. Chandrasena ${ }^{2}$, Mingqiang (Kurt) Gu³, Steven J. May ${ }^{4}$, Burak V. $\mathrm{Ozdol}^{1}$, James Rondinelli ${ }^{3}$, Alexander Gray ${ }^{2}$, and Jim Ciston ${ }^{1}$

${ }^{1}$ National Center for Electron Microscopy, Molecular Foundry, LBNL, CA 94720, USA.

${ }^{2}$ Department of Physics, Temple University, Philadelphia, Pennsylvania 19122, USA

${ }^{3}$ Department of Materials Science and Engineering, Northwestern University, Evanston, IL 60208-3108, USA

${ }^{4}$ Department of Materials Science and Engineering, Drexel University, Philadelphia, Pennsylvania 19104, USA

Exploiting the structural coupling at $\mathrm{ABO}_{3}$ oxide perovskite interfaces has been known as a path for engineering functional properties [1]. By tuning rotations of the corner-connected $\mathrm{BO}_{6}$ octahedra across interfaces, in which the two constituent materials exhibit either a different pattern and/or magnitude of octahedral rotations, new electronic, ferroelectric or magnetic behavior are induced. Consequently, the development and use of new ways of probing subtle changes in the atomic structure and composition simultaneously with the commensurate properties are of great interest as they help defining new routes to tune oxide interfaces.

Recently, the availability of high speed pixelated detectors with reasonable dynamic range have made possible to record full CBED patterns at many probe positions with milliseconds dwell time enabling new transmission electron microscopy (TEM) modes unachievable otherwise [2]. Among these, new experiments that combine rich information from the reciprocal space with precise unit cell localization from scanning TEM (STEM) images are advantageous as they can simultaneously determine composition [3], polarization and octahedral rotation [4] at unit cell resolution.

Here, we apply scanning nanodiffraction methods to investigate modulations of the $\mathrm{MnO}_{6}$ octahedral rotations along the growth direction of isovalent manganite superlattices (SLs), [( $\left.\mathrm{La} 0.7 \mathrm{Sr} 0.3 \mathrm{MnO}_{3}\right) \times 3 /\left(\mathrm{Eu} 0.7 \mathrm{Sr}_{0.3} \mathrm{MnO}_{3}\right) \times$ 3] (namely, LSMO/ESMO) on $\left(\mathrm{La}_{0.3} \mathrm{Sr}_{0.7}\right)\left(\mathrm{Al}_{0.65} \mathrm{Ta}_{0.35}\right) \mathrm{O}_{3}$ (LSAT) substrate. Position averaged CBED (PACBED) [5] are constructed by averaging the probe images over each projected unit cell and compared to a library of previously computed simulated patterns. Sample thickness and octahedral rotation are determined by the best match between the experimental and simulated PACBED images. The limits of precision for quantitatively mapping thickness and octahedral rotation are explored by applying a range of different experimental acquisition parameters [6].

[1] J. M. Rondinelli, S. J. May, and J.W. Freeland, MRS Bull. 37, 261-270 (2012).

[2] C. Ophus, P. Ercius, M. Sarahan, C. Czarnik, J. Ciston, Acta Cryst. A70, C1455 (2014).

[3] C. Ophus, P. Ercius, M. Huijben, and J. Ciston, Applied Physics Letters, 110, p. 063102 (2017).

[4] J. Ciston, et al. Microscopy and Microanalysis 22, p.1412 (2016).

[5] J. M. LeBeau, S. D. Findlay, L. J. Allen, and S. Stemmer, Ultramicroscopy 110, 118 (2010).

[6] Research was performed at the Molecular Foundry, DOE Office of Science User Facility under Contract No. DE-AC02-05CH11231. JC and RdR acknowledge additional support from the DOE Early Career Research Program. 